\title{
Significance of Suitable Analogy Usage to Explain Challenging Theories in Chemistry and
}

Other Fields of Science

Faiz Ahmed*

*Department of Chemistry, Government College University Faisalabad, 38000 Faisalabad, Pakistan.

\section{ABSTRACT}

In elementary chemistry courses students often demonstrate difficulty with real understanding of Resonance Theory i.e. canonical structure vs. real molecule difference, so unanswered puerile questions during lecture made the subject boring. Particularly students unable to understand the difference between a real microscopic moiety and it's proposed sketch or model at early stages of their learning. In such situations use of suitable analogy in front of students other than the subject area make the teaching more effective. Observation through five senses act as a powerful tool to explain curious questions efficiently to develop the interest of the students in subject. Sharing of personal experiences and analogies among scientific community is an effective way to spread scientific knowledge magnificently.

\section{GRAPHICAL ABSTRACT}

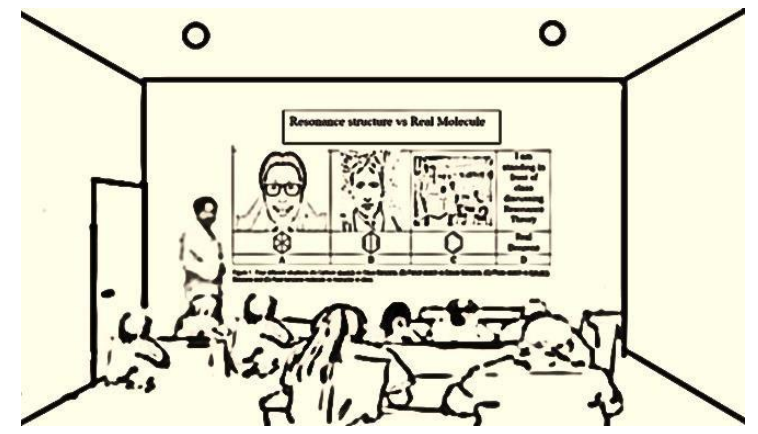

\section{KEYWORDS}

Sketches Analogy, Canonical forms, Real molecule, Resonance Theory, Chemical Pedagogy 
Resonance phenomena understanding play a central role in basic concepts of organic chemistry, however during basic concepts lectures students often demonstrate difficulty with the resonance phenomena (Carle \& Flynn, 2020; Kim, Wright, \& Miller, 2019). Simple questions in undergraduates chemistry class sometime create an environment which force the teacher to think out of the box (Watanabe, Nunes, Mebane, Scalise, \& Claesgens, 2007) to explain these questions using a story (Collins, 2021) or analogy (Shahani \& Jenkinson, 2016) in problem other than the subject i.e. Wheland enthusiast of resonance theory in his book (Wheland, 1944) about resonance phenomena used the analogy of mule which is a hybrid between a horse and a donkey(Wheland, 1944), in an attempt to explain the theories of intermediate stages and of mesomerism picture of the benzene molecule which is a hybrid moiety, not identical with either of the Kekulé's structure or proposed canonical forms but intermediate between them (Strom, 2013). Although resonance theory is one of the most important constituent of structural chemistry (Pauling, 1977), still highly useful and important section of the pedagogy of organic chemistry concepts (Herndon, 1973) however in some situations this use of imaginary institution to describe real molecules was incompatible with the dialectical materialism (Strom, 2013; Wheland, 1944, 1948). Similar type of problem faced by the organic chemist while explaining the structure of organic compounds especially the canonical forms and the actual molecule while discussing the Rules of resonance (Smith, 2019). During basic chemistry courses all over the world a small fraction of students raise puerile questions during the lecture (Orvis et al., 2016), which are ignored by the teachers sometime to save the lecture time and utility on more important concepts as a result that small fraction of students find chemistry as boring subject, where imaginary things are discussed and taught. However suitable analogy related to the query or topic make it easy (Cortés-Figueroa, Pérez, López, \& Moore-Russo, 2011), understandable and develop students interest in the subject (Orgill, Bodner, Pienta, Cooper, \& Greenbowe, 2005; Silverstein, 1999; Spezzini, 2010) i.e. cartoon characters analogy for resonance by R. Starkey (Starkey, 1995). 
Explanation of Canonical Structure Vs. Real Molecule in Resonance Phenomena: 
During my 15 years teaching experience in organic chemistry, I also met with students who find difficult to understand such lines "All canonical forms do not contribute equally to the true molecule and it is more stable than all these canonical forms" (Smith, 2019) and they immediately raise the questions, what is real molecule and why we are unable to draw its exact structure? In start of my career I also used Wheland analogy (Strom, 2013) however students curiosity remain unsatisfied, sometime few raise objection that mule, donkey and horse are three different animals and all has existence, while here we are discussing only one molecule that is benzene. After the flash of these questions more students join that small fraction of students with doubts. To face such situation I developed following questions and sketch analogy.

Question: Class can we represent a person in any discussion or story by writing his name or using his photo?

Class Answer: Yes we can represent a person by writing his name.

Question: In chemistry how we can represent a chemical substance?

Class Answer: Through its name, chemical formula, CAS Registry Number or draw its structure to discuss it.(Sometime I also participate in this answer)

Next we discussed about organic compounds simple chemical formula from which different structures can be deduced i.e. ethanol and dimethyl ether both have same chemical formula $\mathrm{C}_{2} \mathrm{H}_{6} \mathrm{O}$, to overcome this issue organic chemist prefer to represent each compound with localized bonding systems through Lewis structure, while compounds with delocalized bonding system are represented through different canonical forms using Resonance theory or method.

\section{Sketch Analogy:}


After these question answers and discussion I draw few sketches about myself on white/black board or show my photos or emojis on screen according to the situation along with different structure of benzene proposed by different scientists (See Figure 1).

After showing Figure 1, I ask the students following questions:

Question: Is column A, B and C photos in instructor's sketch row represents me?

Answer: Yes.

Question: In current situation which photo/sketch represents me most and which on least?

Answer: In instructors sketch row, photo in column $\mathbf{C}$ represents me more while $\mathbf{A}$ represents least.
Instructor's sketch
A

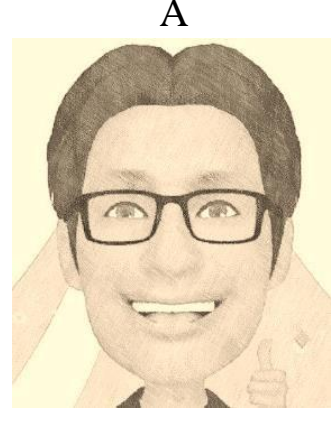
B
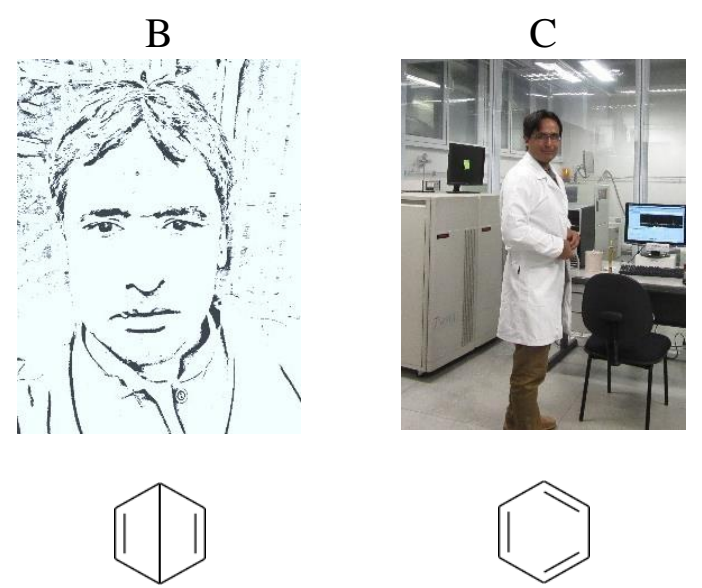
D I am standing in front of class discussing Resonance Theory
Real benzene molecule is in reagent bottle on the desk.

Figure 1. Four different situations (A) Cartoon sketch vs. Claus Benzene, (B) Pencil sketch vs. Dewar Benzene, $(\mathbf{C})$ Photo sketch vs. Kekule's Benzene and (D) Real benzene molecule vs. Instructor in class.

Then I continue that even $\mathbf{C}$ does not represent me completely because it only represent my side which is in front of camera, it also does not move as I am moving in front of whole class, which create fourth situation D my real existence. Due to limitations of two dimensional sketch on paper it is not possible to create a duplicate copy of a person on a paper. Similarly, same case is with benzene canonical structure in column $\mathbf{A}, \mathbf{B}$ and $\mathbf{C}$, however these canonical structures provide us an opportunity to discuss a molecule on a paper, on board or in a book. Although we 
are unable to draw a real benzene molecule which is actually in the reagent bottle on the desk which also creates fourth situation $\mathbf{D}$, however by following resonance rules we can draw its different possible sketches to represent and discuss it and this discussion is based on different evidences, which are further divided into two different types direct evidence and indirect evidence. Direct evidence is that evidence which we perceive through our five senses, while indirect evidence is with the help of an instrument i.e. microscope, electron microscope, camera or measurement of a physical parameter or phenomena. In chemistry we use different instrument like nuclear magnetic resonance or mass spectrometer etc. which give us indirect evidence about the existence of atoms and their linkages in molecules. On the basis of these pieces of evidence we draw a model or structure to represent molecules. Just like my sketches or photos on screen which are indirect evidences about my existence while my presence in front of you is my real existence. Similarly we draw molecular or atomic models on paper or board are on the basis of indirect evidences about respective molecules and atoms which also has existence, however we are unable to see even they are in front of us due to limitation of our five senses. As visible light spectrum consist of seven colours which can be observed when sunlight is passed through a prism. Similarly benzene which is in liquid state also consist of small molecules which we are unable to see with our five senses doesn't mean they don't have existence. So scientist built it's model on the basis of indirect evidences obtained through different instruments and techniques. After this explanation I ask whole class did you understand the canonical forms and a real molecule. They say yes and have no further doubts.

\section{Methodology to Check Macro entity and its Sketch Analogy Effectiveness:}

To check the effectiveness of above analogy, conduct the short quiz survey among three different groups of 15 - 30 students class, studying chemistry in final years of their levels, Group A twelve year education, Group B sixteen year education and Group C eighteen year education, collected 150 students response on average from each group summarized the conclusion of quiz survey in the form of graph in Figure 2. 
Table 1: Quiz before and after Discussion

Quiz survey before discussion Time 10 min
\begin{tabular}{|r|l|l|l|}
\hline 1. Choose the Correct Option & Yes & NO \\
\hline i). & Structure drawn on the Paper are the real molecules. & Yes \\
\hline ii). & $\begin{array}{l}\text { Structure drawn on the paper are the sketches or representation of } \\
\text { real molecules. }\end{array}$ & Yes & NO \\
\hline iii). & Real molecule has existence. & Yes & NO \\
\hline iv). & Each canonical structure or form of a molecule has existence. & Small size & $\begin{array}{l}\text { Non } \\
\text { existence }\end{array}$ \\
\hline v). & Humans unable to see real molecular structure due to &
\end{tabular}

2. Explain real molecule and its canonical structure in three to four lines.

Quiz survey after discussion Time $10 \mathrm{~min}$

1. Choose the Correct Option

i). Real molecules can be represented through structure drawn on the Paper.

ii). Canonical structures are the ways to discuss a real molecule's structure.

iii). We are unable to see a real molecule so it has no defined structure.

iv). There is no need to discuss canonical structures of a molecule in resonance.

v). Only visible substances have proper structure.

vi). Different structure of benzene are its canonical forms while real molecule is more stable than all of these.

2. Explain real molecule and its canonical structure in three to four lines.

\section{\%age of students with their understanding level before and after discussion}

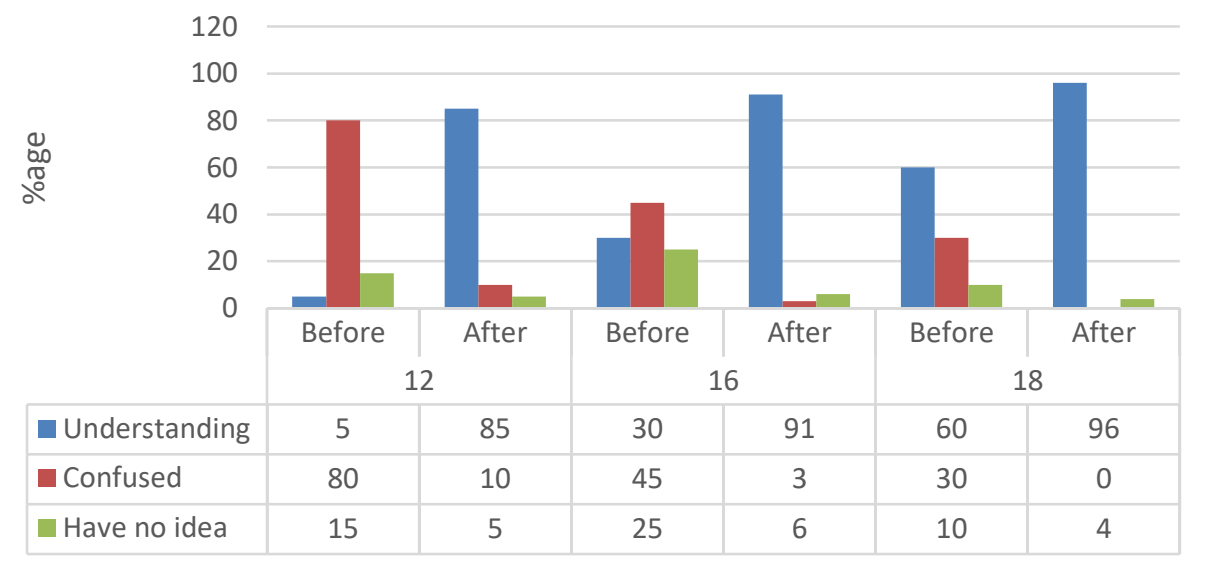

Figure 2 Conclusion of students response Understanding: Students can clearly explain the real molecule and its canonical structures, Confused : Students have confusion between real molecule and its canonical structures, Have no Idea: Student have no knowledge or no idea about real molecule or did not responded. Standard error : \pm 1.4 


\section{Conclusion :}

Views and downloads at https://chemrxiv.org/engage/chemrxiv/author-dashboard after submission of above discussed content proves that real molecule structure and its canonical forms explanation still searched by the chemist and chemistry students. Thus a simple analogy act as a powerful tool to develop the interest of students in subject with better elucidation, so there is need to share more such personal experiences and analogies to spread scientific knowledge more effectively all around the world. We can also use any other living organism sketches i.e. dog, cat etc. as an example, however instructor's sketches and presence in front of class act as an influential instrument for students understanding toward the difference between canonical structure and real molecule.

\section{AUTHOR INFORMATION}

Corresponding Author

*E-mail: faiz79goraya@gmail.com, faizahmed@gcuf.edu.pk

\section{REFERENCES}

Carle, M. S., \& Flynn, A. B. (2020). Essential learning outcomes for delocalization (resonance) concepts: How are they taught, practiced, and assessed in organic chemistry? Chemistry Education Research and Practice, 21(2), 622-637. doi:10.1039/C9RP00203K

Collins, S. N. (2021). The importance of storytelling in chemical education. Nature Chemistry, 13(1), 1-2. doi:10.1038/s41557-020-00617-7

Cortés-Figueroa, J. E., Pérez, W. I., López, J. R., \& Moore-Russo, D. A. (2011). An Analogy Using Pennies and Dimes To Explain Chemical Kinetics Concepts. J. Chem. Educ., 88(7), 932.

Herndon, W. C. (1973). Resonance energies of aromatic hydrocarbons. Quantitative test of resonance theory. Journal of the American Chemical Society, 95(7), 2404-2406. doi:10.1021/ja00788a073

Kim, T., Wright, L. K., \& Miller, K. (2019). An examination of students' perceptions of the Kekulé resonance representation using a perceptual learning theory lens. Chemistry Education Research and Practice, 20(4), 659-666. doi:10.1039/C9RP00009G

Orgill, M., Bodner, G., Pienta, N., Cooper, M., \& Greenbowe, T. (2005). Chemists' Guide to Effective Teaching.

Orvis, J., Sturges, D., Rhodes, S., White, K.-J., Maurer, T. W., \& Landge, S. M. (2016). A Mailman Analogy: Retaining Student Learning Gains in Alkane Nomenclature. Journal of Chemical Education, 93(5), 879-885. doi:10.1021/acs.jchemed.5b00551

Pauling, L. C. (1977). The theory of resonance in chemistry. Proceedings of the Royal Society of London. A. 
Mathematical and Physical Sciences, 356(1687), 433-441. doi:doi:10.1098/rspa.1977.0143

Shahani, V. M., \& Jenkinson, J. (2016). The efficacy of interactive analogical models in the instruction of bond energy curves in undergraduate chemistry. Chemistry Education Research and Practice, 17(2), 417-428. doi:10.1039/C5RP00194C

Silverstein, T. P. (1999). The "Big Dog-Puppy Dog” Analogy for Resonance. J. Chem. Educ., 76(2), 206.

Smith, M. B. (2019). Delocalized Chemical Bonding. In March's Advanced Organic Chemistry (pp. 32-105).

Spezzini, S. (2010). Effects of Visual Analogies on Learner Outcomes: Bridging from the Known to the Unknown. Int. J. Scholarship Teach. Learn., 4(2), 1.

Starkey, R. (1995). Resonance Analogy Using Cartoon Characters. J. Chem. Educ., 72(6), 542.

Strom, E. T. (2013). George W. Wheland: Forgotten Pioneer of Resonance Theory. In Pioneers of Quantum Chemistry (Vol. 1122, pp. 75-115): American Chemical Society.

Watanabe, M., Nunes, N., Mebane, S., Scalise, K., \& Claesgens, J. (2007). "Chemistry for all, instead of chemistry just for the elite": Lessons learned from detracked chemistry classrooms. Science Education, 91(5), 683-709. doi:https://doi.org/10.1002/sce.20213

Wheland, G. W. (1944). The Theory of Resonance and its Application to Organic Chemistry.

Wheland, G. W. (1948). Advanced Organic Chemistry. 